\title{
0 Envolvimento de idosos em atividades prazerosas: Revisão da Literatura sobre Instrumentos de Aferição
}

Elderly people's involvement in pleasant activities: a Literature Review on Measurement Instruments

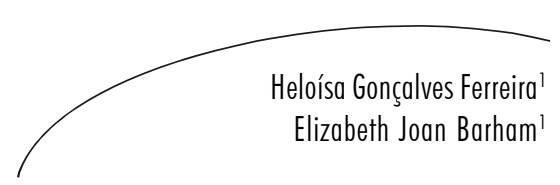

Resumo

O prazer experimentado ao se envolver em atividades é fator fundamental para o bem-estar do idoso. Estudos mostram que prática de atividades agradáveis atua como variável mediadora na prevenção e superação de desordens psicológicas, ajuda a lidar com os efeitos negativos de perda de funcionalidade, viuvez e pouco contato familiar, bem como prediz bem-estar físico e psicológico. A literatura consultada mostrou que, no Brasil, as pesquisas que abordam esse tema são incipientes, e que não existe ainda um instrumento validado para a realidade brasileira que avalie envolvimento de idosos em atividades prazerosas. O objetivo deste estudo foi comparar instrumentos existentes que avaliam a frequência do envolvimento de idosos em atividades e o nível de prazer subjetivo experimentado em cada uma delas, para então sugerir um instrumento a ser submetido a um processo de validação transcultural no Brasil. As bases de dados utilizadas foram: CAPES, SciElo, LILACS e Medline. Não foram localizados instrumentos brasileiros que medissem o envolvimento de idosos em atividades prazerosas. Foram localizados três instrumentos internacionais: The California Older Person's Pleasant Events Schedule (COPPES); The Pleasant Events Schedule-AD (PES-AD) e The Pool Activity Level (PAL). Todos os instrumentos apresentaram boas propriedades psicométricas, no entanto o COPPES aparentou ser o instrumento mais apropriado para uma validação transcultural para o Brasil. Espera-se que um instrumento desta natureza possa contribuir para a realização de pesquisas, planejamento e avaliação de intervenções e estratégias preventivas focadas na promoção de bemestar entre idosos, no Brasil.

\footnotetext{
Programa de Pós-Graduação em Psicologia da Universidade Federal de São Carlos. São Carlos, SP, Brasil. E-mail: helogf@gmail.com

Este artigo é parte da dissertação da primeira autora, orientada pela segunda, no Programa de PósGraduação em Psicologia da UFSCar (São Carlos - SP - Brasil), e foi apoiado com Bolsa de Mestrado da Fundação de Apoio à Pesquisa do Estado de São Paulo (FAPESP, Processo no. 08/ 56855-6).
}

Palavras-chave: Idoso. Atividades Prazerosas. Satisfação Pessoal. Qualidade de vida. Instrumentos Psicométricos. Validação Transcultural. 


\section{Abstract}

The elderly pleasure in engaging in activities is an essential factor for their wellbeing. Investigations show that the practice of pleasant activities works as an intermediate variable in preventing and coping with psychological disorders, negative effects of functionality loss, widowhood and lack of familiar contact, as well as predict physical and psychological well-being. The literature shows that, in Brazil, investigations about this field are still incipient, and there are not validated instruments which measure the involvement of Brazilian elderly in pleasant activities. The aim of this study was to review the literature to identify and compare instruments which measure the frequency of elderly people's involvement in pleasant activities, and then suggest an instrument to be submitted to a cross-cultural validation process in Brazil. The searched databases were: CAPES, SciElo, LILACS and Medline. We found no Brazilian instruments that measure the elderly's involvement in pleasant activities. They were found three international instruments: The California Older Person's Pleasant Events Schedule (COPPES); The Pleasant Events Schedule-AD (PES-AD) and The Pool Activity Level (PAL). All instruments had good psychometric properties, but COPPES was the one which sounded the most appropriate for a cross-cultural validation process in Brazil. An instrument of this kind may contribute to further researches, planning and evaluations of interventions and preventive strategies focused in promoting elderly people's well-being in Brazil.

\author{
Key words: Elderly. \\ Pleasant Activities. \\ Personal Satisfaction. \\ Quality of life. \\ Psychometric Instruments. \\ Cross-Cultural Validation.
}

\section{INTRODUÇÃO}

A velhice, embora seja uma conquista, traz ao mesmo tempo o desafio de descobrir como pode ser desfrutada. Assim, a questão de como ocupar o tempo de forma satisfatória nessa fase, tanto no nível individual quanto social, é de fundamental importância. A literatura permite verificar que existe relação entre depressão entre idosos e a baixa frequência de envolvimento em atividades. ${ }^{1-8}$ Para prevenir ou tratar este problema, é necessário obter informações sobre como aumentar a probabilidade de o idoso se manter ativo. Alguns estudos afirmam ser essencial considerar o prazer subjetivo do idoso ao se envolver em atividades. ${ }^{9,10}$

No Brasil não existem instrumentos para aferir o envolvimento de idosos em atividades ou eventos que geram sentimentos positivos. Por isso, este estudo tem por objetivo contribuir com a validação transcultural de um instrumento para avaliar a frequência de envolvimento de idosos em eventos prazerosos. Para demonstrar a importância de contar com um instrumento desta natureza, este trabalho primeiramente traz uma revisão da literatura sobre teorias de atividade e pesquisas que relacionam a prática de atividades a sentimentos positivos na velhice.

No censo de 2008, o crescimento da população idosa brasileira representou mais que o dobro do crescimento da população total, indicando que o Brasil caminha velozmente rumo a um perfil demográfico cada vez mais envelhecido. A estimativa é de que a vida média do brasileiro chegará ao patamar de 81 anos, em 2050. ${ }^{11}$

Diante do aumento da população idosa brasileira, torna-se cada vez mais clara a importância de buscar alternativas para permitir um envelhecimento o mais saudável e bemsucedido possível. Pesquisas sobre condições que permitem uma qualidade de vida satisfatória para idosos e que consideram diferentes trajetórias do processo de envelhecimento ${ }^{6-8,12-14}$ estão contribuindo para identificar possíveis maneiras de prevenir e tratar alguns problemas de saúde 
física ou mental, como por exemplo, a depressão, nesta população. Um fator que vem ganhando destaque em relação à questão do envelhecimento bem-sucedido é o envolvimento de idosos em atividades que geram sentimentos positivos para os mesmos.

A associação entre atividade e bem-estar na velhice tem sido alvo de pesquisas e formulações teóricas na literatura gerontológica. A investigação sistemática sobre o ajustamento pessoal e social na velhice foi iniciada em meados da década de 40 , sendo que na década de 50 os pesquisadores começaram a enfatizar a importância da atividade como um dos componentes indispensáveis para um envelhecimento bem-sucedido. ${ }^{15}$

Atividade é um conceito-chave relativamente recente que tem ajudado os gerontólogos a compreenderem o processo de envelhecimento. No entanto, o termo "atividade" tem sido conceitualizado, categorizado e mensurado de muitas formas, a depender $\mathrm{da}$ área de pesquisa $\mathrm{e}$ de intervenção.

Não existe uma definição geral ou uma ciência $\mathrm{da}$ atividade que integra todas as definições mais específicas existentes nos diferentes campos. ${ }^{15} \mathrm{O}$ que existem são diferentes profissionais interessados em investigar ou intervir em contextos envolvendo diversos tipos de atividade. Por exemplo, alguns pesquisadores estudam atividades restritas ao domínio físico (movimentos, atividades e exercícios físicos); ${ }^{16-19}$ outros estudam atividade no sentido de busca de interesses diários e investigam atividade no que diz respeito à participação social; ${ }^{15}$ outros ainda definem atividade como sinônimo de ocupação do tempo, podendo incluir atividades físicas, mentais, individuais e grupais. ${ }^{9}$ Outros autores ${ }^{20-23}$ também consideram que atividade se relaciona a ocupação de tempo e definem atividade de acordo com a forma com que os indivíduos utilizam seu tempo. Além disso, tais formas de atividade podem ser estudadas tanto juntas como separadas.

No decorrer dos anos 60, o número de pesquisas sobre a relação entre atividade e satisfação cresceu, em decorrência do aumento da demanda social para informações a este respeito, ligada ao crescimento da população idosa, principalmente, nos EUA e Europa. A partir de 1970, começou a ser postulado que a atividade poderia ser um determinante da satisfação, uma vez que permitia que o indivíduo experimentasse um domínio sobre o ambiente e sobre os eventos de sua vida. ${ }^{24}$

A Teoria da Atividade formulada em 1953 por Havighurst, se propõe a explicar a relação entre atividade e satisfação na velhice. ${ }^{25}$ Esta teoria assume que quanto mais ativo for o idoso, maior será sua satisfação na vida. Idosos com interações sociais mais frequentes e que desempenham as atividades esperadas para o seu grupo etário tendem a gerar aprovação social que, por sua vez, promove um autoconceito positivo e resulta em maior satisfação na vida. De acordo com esta teoria, o envelhecimento pode ser uma experiência criativa e saudável, sendo que seria a inatividade, e não o aumento da idade, que levaria ao declínio.

A Teoria da Atividade foi adotada e reformulada por Rowe $\& \mathrm{Kahn},{ }^{26}$ que enfatizaram a importância de evitar doenças, manter o funcionamento físico e cognitivo, além de estabelecer relações próximas para garantir um bom envelhecimento. Evitando doenças, tornase mais fácil envolver-se em atividades sociais e de lazer e, consequentemente, evitar isolamento social e declínio físico.

Silverstein \& Parker $^{27}$ também tomam por base a Teoria da Atividade para investigar o bem-estar de idosos e acrescentaram a ideia de que é importante o idoso se adaptar às novas condições da velhice, por meio de uma substituição e redistribuição de atividades. Estes autores afirmam existir um declínio em atividades de lazer com o passar do tempo, e por isso é importante que exista aumento na variabilidade dos tipos de atividades que são praticadas. Aumentar a variedade de categorias de atividades ou evitar a sua perda contribuiria para a qualidade de vida do idoso. $\mathrm{O}$ envolvimento em atividades sociais e de lazer em ambientes diversificados e que envolvem a interação com outras pessoas também seria importante para predizer bem-estar físico e psicológico. ${ }^{14}$ 
Para outros autores, é importante atentar não apenas para indicadores da quantidade de envolvimento em atividades, mas também para a importância de aspectos relacionados à atividade que levam o indivíduo a experimentar satisfação., ${ }^{9,10}$ Nesta perspectiva, manter-se em atividade só será uma maneira do idoso manter seu bem-estar físico e mental, caso traga significado e satisfação à existência, quer pelo compromisso e responsabilidade social implícitos na atividade, quer pela oportunidade de manter o convívio social. Ou seja, só há significado em atividades que envolvam objetivos que tragam satisfação, uma vez que sem satisfação subjetiva, o significado é incompleto.

Existem diversos estudos que apoiam a ideia de que atividades que proporcionam satisfação afetam a saúde do indivíduo. Por exemplo: (a) a prática de atividades prazerosas pode atuar como variável mediadora na superação e prevenção do estresse, depressão e outras desordens psicológicas em idosos; ${ }^{6,7,9,12}$ (b) ajuda a lidar com os efeitos negativos da perda de funcionalidade, viuvez e pouco contato familiar; ${ }^{27}$ (c) associa-se à expressão de afeto positivo; ${ }^{6,21,28}$ e (d) afeta o nível de socialização e interesse pela vida. ${ }^{12}$

\section{PRÁTICA DE ATIVIDADES PRAZEROSAS E DEPRESSÃO NA VELHICE}

As desordens mentais comprometem $20 \%$ da população idosa, entre as quais se destaca a depressão. No Brasil, aproximadamente 10 milhões de idosos sofrem de depressão. ${ }^{29} \mathrm{~A}$ depressão é o transtorno psiquiátrico mais frequente entre indivíduos com idade avançada. ${ }^{30}$ Devido à prevalência da depressão na população idosa, a relação entre envolvimento em atividades prazerosas e esta desordem psicológica é um tópico que necessita ser estudado em detalhes, para buscar compreender como essas variáveis se relacionam.

Um modelo que busca explicar a relação entre depressão e prática de atividades prazerosas é o modelo comportamental proposto por Lewinsohn \& Libet. ${ }^{1}$ De acordo com esse modelo, a depressão é causada por uma baixa taxa de respostas capazes de gerar reforçadores positivos, ou seja, indivíduos depressivos não geram respostas suficientes em seu ambiente para gerar reforços positivos. No caso, reforçadores positivos são definidos como eventos positivos experenciados de forma subjetiva, ou seja, eventos que o indivíduo avalia como sendo prazerosos. ${ }^{6}$

Lewinsohn \& Libet constataram existir associação significativa entre a prática de atividades prazerosas e o estado de humor, que é consistente com a teoria comportamental sobre depressão que afirma existir associação entre grau de reforçamento positivo (em função do envolvimento em atividades prazerosas) e intensidade de depressão. ${ }^{1} \mathrm{Ou}$ seja, quanto mais um indivíduo estiver engajado em atividades prazerosas, melhor será seu estado de humor, pois o engajamento em eventos ou atividades consideradas prazerosas pelo indivíduo resulta em consequências positivas para o mesmo. Quanto menos o indivíduo estiver engajado em atividades prazerosas, menos consequências positivas e maior chance de experimentar desânimo ou até evoluir para depressão.

O prazer tem sido identificado como um fator fundamental para o bem-estar, abrindo a perspectiva de que não apenas as atividades de lazer, mas qualquer atividade relacionada com o prazer, pode ter efeitos positivos na saúde mental. Tomando por base esta ideia, não é a atividade propriamente dita que seria positiva ou não para a saúde mental do indivíduo, mas sim as consequências obtidas por meio do envolvimento na atividade, como por exemplo, o prazer que o indivíduo experimenta ao realizá-la. Elevada concentração, perda da noção do tempo, experiência intrínseca de recompensa e um equilíbrio entre desafio e senso de domínio são alguns dos fatores considerados como sendo responsáveis pelo prazer trazido pela prática de atividades. ${ }^{10}$

Desta forma, um aspecto muito importante a ser considerado é o interesse do idoso em praticar ou não determinado tipo de atividade, incluindo atividades novas. Por esse motivo, descobrir as 
preferências dos idosos e dar-lhes controle para que escolham o que fazer é imprescindível para que os benefícios da prática de atividades sejam observados. ${ }^{6,15}$ No entanto, é essencial considerar a concepção que os próprios idosos constroem sobre a importância de atividade na velhice (valorizar ser mais ativo ou menos ativo em determinadas áreas). Tal consideração deve ser feita não com base em teorias gerontológicas ou programas de atividades, mas com base nas expectativas que o próprio idoso tem para esta fase da vida e nas experiências vivenciadas por ele nos ambientes de seu cotidiano. ${ }^{15}$

Além dos efeitos de envolvimento em atividades prazerosas para idosos depressivos, existem estudos investigando os impactos da prática de atividades prazerosas em idosos depressivos com doença de Alzheimer. ${ }^{3,8,21,31}$ Idosos com doença de Alzheimer (DA) experimentam uma perda gradual das habilidades para desempenharem atividades que lhes sejam gratificantes e agradáveis, em função do declínio cognitivo característico da doença. A falta de atividades prazerosas pode exacerbar os prejuízos cognitivos associados à doença de Alzheimer. Ao mesmo tempo, seguindo a lógica já descrita, percebe-se que a incapacidade de realizar atividades prazerosas que aparece entre idosos com DA também aumenta a probabilidade de estes desenvolverem depressão. Idosos e cuidadores que precisam lidar com a demência e a depressão ficam duplamente sobrecarregados. Por isso, identificar atividades prazerosas que idosos dementados possam praticar ajuda a aliviar a depressão, ao mesmo tempo em que provê benefícios individuais e interpessoais, aumentando a qualidade do contato social entre o idoso e os demais. ${ }^{3}$

Um estudo americano constatou existir relação entre eventos considerados como sendo prazerosos pelo cuidador e pelo idoso, funcionamento cognitivo e depressão do idoso. ${ }^{4}$ Participaram desse estudo 42 cuidadores de idosos com DA (não-institucionalizados) que responderam, juntamente com o idoso, a uma escala que avaliava: frequência, disponibilidade e o quão agradável foi envolver-se em atividades potencialmente agradáveis durante o último mês.
Constatou-se que frequência e disponibilidade de atividades não apresentaram relação com depressão ou status cognitivo. O que demonstrou relação com depressão foi a frequência de atividades que o paciente relatou gostar de fazer, indicando a importância do prazer subjetivo relacionado à prática de atividades. Além disso, menos atividades prazerosas foram reportadas por indivíduos depressivos se comparados aos nãodepressivos, independentemente do status cognitivo. Esse resultado corroborou a hipótese de que depressão está relacionada com o envolvimento em atividades prazerosas, inclusive em indivíduos com comprometimento cognitivo.

Outro estudo ${ }^{6}$ constatou que atividades padronizadas em instituições para idosos podem não ser efetivas no sentido de gerar prazer para todos os participantes. Ou seja, existem diferenças individuais com relação aos tipos de eventos que são prazerosos para cada idoso. Neste estudo, as atividades relacionadas ao interesse dos participantes foram as mais importantes para aliviar sintomas de depressão nos pacientes institucionalizados.

É importante respeitar e considerar a subjetividade do idoso para não correr o risco de equacionar o envelhecimento bem-sucedido com uma "receita pronta", na qual se manter ativo seria o principal ingrediente. ${ }^{15} \mathrm{E}$ imprescindível que variáveis como contexto cultural, história de vida do idoso e sentimentos experimentados pelo mesmo sejam levadas em conta. Estes fatores permitem compreender o significado que cada atividade tem para esse indivíduo, bem como, o ritmo de vida que lhe seja agradável em seu dia a dia.

A prática de atividades prazerosas por idosos não tem recebido atenção especial no Brasil. Com relação a atividades de lazer para idosos, faltam ainda políticas públicas e sociais voltadas para esta questão. $\mathrm{O}$ acesso às oportunidades de lazer é restrito para a maioria dos idosos por diversos motivos, tais como: restrições financeiras, barreiras arquitetônicas para idosos com dificuldades de locomoção, necessidade de ajuda para sair de casa, além da falta de temas do interesse para esta população. Além da falta de 
políticas públicas, no âmbito científico, são escassos os trabalhos que enfoquem a contribuição do lazer para a saúde e qualidade de vida na população idosa brasileira. ${ }^{12}$

Com o crescimento da população idosa brasileira, surge o desafio de implantar novos modelos e métodos para atender a esse tipo de população, de forma a garantir sua qualidade de vida. Neste sentido, é importante contar com instrumentos psicométricos que avaliem o envolvimento de idosos em atividades que lhes sejam prazerosas. É importante valer-se de diferentes estratégias para acompanhar e registrar o desenvolvimento de cada idoso, por exemplo, utilizando instrumentos validados que auxiliem no entendimento da demanda de cada pessoa e na compreensão de sua subjetividade. ${ }^{13}$ As informações obtidas por meio desses instrumentos também poderiam contribuir para o planejamento e avaliação de intervenções que visem a promover o bem-estar dos idosos. Estudos sobre a prática de atividades que sejam prazerosas para o idoso poderiam também ajudar a desmistificar a crença vigente entre leigos e profissionais, da velhice associada à deterioração e à inatividade.

Considerando a prevalência de depressão na população idosa brasileira, os benefícios associados à prática de atividades prazerosas e os dados da literatura que sugerem existir associação entre prática de atividades prazerosas e estado de humor em idosos, torna-se extremamente relevante compreender e pesquisar mais profundamente este assunto na população brasileira, para que essa morbidade seja diagnosticada e tratada d modo adequado. Para tal, é necessário contar com escalas validadas na língua portuguesa, com boas propriedades psicométricas e adaptadas ao contexto brasileiro.

Logo, este estudo tem por objetivo realizar uma revisão de literatura para identificar e descrever instrumentos psicométricos que avaliem o envolvimento de idosos em atividades prazerosas, para indicar um instrumento para ser submetido a um processo de validação transcultural no Brasil.

\section{METODOLOGIA}

Foi realizada busca bibliográfica, em 2009, nas seguintes bases de dados: CAPES, SciELO, LILACS e Medline. Foram utilizadas as seguintes palavras-chave em português: idoso ou envelhecimento, cruzado com atividade; atividades; atividades prazerosas; prazer; atividades de lazer; lazer e com instrumento; escala; confiabilidade; validade; e em inglês: elderly ou olderperson cruzado com activity; activities; pleasurable activities; pleasant; pleasant events; leisure activities e com instrument; scale; validity. Não houve limitação de data e foram considerados para análise apenas artigos de livre acesso.

\section{RESULTADOS E DISCUSSÃO}

Foram encontrados 76 artigos, sendo 19 referentes a instrumentos psicométricos. Desses 19 artigos, foram excluídos 16 artigos sobre instrumentos psicométricos que avaliavam construtos que não correspondem ao foco deste estudo, tais como: estado funcional do idoso (atividades básicas, instrumentais ou avançadas da vida diária), atividade física, movimento físico e qualidade de vida. Esses instrumentos levantam a capacidade do idoso para realizar diversas tarefas (funcionalidade) ou o envolvimento do idoso em diferentes tipos de atividade. No entanto, estas medidas não avaliam o grau de prazer que o idoso sente ao realizar as atividades.

Foi encontrado um estudo brasileiro ${ }^{22}$ que utilizou a adaptação de uma escala australiana ${ }^{20}$ que avalia a frequência e a duração das atividades humanas. Essa escala foi aplicada em uma amostra de 75 idosas brasileiras com o objetivo de descrever de que forma as participantes utilizavam seu tempo. Entretanto, a escala adaptada utilizada neste estudo não avalia o prazer que o idoso experimenta ao praticar a atividade. Por este motivo, o instrumento não foi considerado para análise, uma vez que o objetivo da revisão era localizar instrumentos que avaliavam tanto a frequência da prática de atividades por idosos, quanto o prazer subjetivo experimentado na prática das atividades. Não foram localizados, 
assim, instrumentos brasileiros que medissem o envolvimento de idosos em atividades prazerosas. $\mathrm{Na}$ literatura internacional, foram encontrados três instrumentos que atendiam aos critérios de inclusão. ${ }^{3,5,31}$ Esses instrumentos serão descritos detalhadamente a seguir.

(1) The Pleasant Events Schedule - AD: escala americana com 53 itens que descrevem eventos e atividades para idosos com doença de Alzheimer. As atividades descritas foram selecionadas com base na experiência clínica dos autores, junto a idosos com doença de Alzheimer. As atividades foram escolhidas para cobrirem duas dimensões primárias: (1) atividades passivas e ativas; $\mathrm{e}$ (2) atividades sociais e não-sociais. Os itens estão de acordo com as habilidades cognitivas de idosos portadores de demência moderada, sendo que algumas exigem maior grau de habilidade cognitiva (ex: montar quebracabeças) do que outras (ex: escutar música). A escala mensura frequência e disponibilidade das atividades e o quão agradável foi envolver-se em cada uma delas durante o último mês. Frequência e disponibilidade são mensuradas numa escala ordinal em que as opções de respostas são as seguintes: 0 = "nunca"; 1 = "algumas vezes"; e 2 = "frequentemente”. A satisfação em realizar a atividade é avaliada com duas opções de respostas: (0) se foi agradável realizar a atividade no passado; e (1) se é agradável realizar a atividade atualmente. A frequência com a qual a atividade é praticada e a satisfação atual com a atividade são multiplicadas para produzir um escore para cada item. Os escores de todos os itens são somados para produzir o escore total para o teste, que representa a frequência de envolvimento em atividades prazerosas durante o último mês.

(2) The California Older Person Pleasant Events Schedule-COPPES: instrumento americano composto por 66 itens que descreve atividades que idosos tendem a achar prazerosas. $O$ respondente deve classificar em "nunca", "1-6 vezes" ou "mais de sete vezes", a frequência com a qual esteve envolvido em cada atividade no último mês. O respondente também deve classificar o prazer subjetivo que experimentou ao realizar cada atividade, ou que experimentaria caso tivesse realizado, tendo como opções de classificação "não foi ou não teria sido agradável”, "foi ou teria sido razoavelmente agradável" e "foi ou teria sido bastante agradável”. O instrumento foi inicialmente construído para ser utilizado em contexto clínico para tratar idosos depressivos, tendo por base o modelo comportamental de depressão de Lewisohn (1972). No entanto, o instrumento também pode ser aplicado em idosos não-depressivos, em outros contextos onde seja relevante aferir a frequência de envolvimento em atividades prazerosas do idoso. As atividades descritas no instrumento são divididas em cinco dimensões: (1) socialização; (2) relaxamento; (3) contemplação; (4) ser efetivo; e (5) ser ativo.

(3) The Pool Activity Level Checklist - PAL: instrumento desenvolvido para identificar o nível de habilidade cognitiva que um idoso dementado precisa ter para ser capaz de engajar-se em atividades associado com informações sobre o histórico de preferências do idoso para realizar diferentes atividades. Quem responde ao instrumento é o cuidador primário do idoso. O modelo teórico que fundamentou sua construção foi the Functional Information Processing Mode $\beta^{1}$. O instrumento é sempre aplicado em associação com um segundo instrumento, o qual é usado para levantar o perfil de interesses do idoso - Personal History Profile. Ambos as medidas devem ser preenchidas para auxiliar o cuidador a escolher atividades significativas e acessíveis ao idoso, de acordo com seu grau de comprometimento. No PAL, são avaliadas quais as atividades que o idoso ainda é capaz de realizar, agrupadas em quatro níveis de complexidade: atividades planejadas, atividades exploratórias, atividades sensórias e atividades passivas (por exemplo, 
receber massagem, escutar música). $\mathrm{O}$ instrumento classifica as atividades cotidianas em nove categorias: (1) tomar banho/lavar-se; (2) vestir-se; (3) comer; (4) contato com outras pessoas; (5) habilidades para trabalhar em grupo; (6) habilidades de comunicação; (7) atividades práticas (ex: atividades domésticas, jardinagem, artesanato); (8) uso de objetos e (9) olhar jornais/revistas. Dentro de cada categoria de atividade, existem quatro sentenças descritivas do grau de habilidade do idoso. $O$ cuidador deve assinalar qual sentença melhor descreve o desempenho do idoso, considerando as duas últimas semanas. Com base nestas informações, o nível de atividade em que o idoso se encontra é então aferido.

As principais informações sobre identificação desses instrumentos encontram-se na tabela 1.

Tabela 1 - Identificação dos instrumentos que avaliam o envolvimento de idosos em atividades prazerosas. São Carlos, SP, 2009.

\begin{tabular}{|c|c|c|c|c|c|c|c|}
\hline Instrumento & O que avalia & $\begin{array}{l}\text { Quem é } \\
\text { avaliado }\end{array}$ & Respondente & $\begin{array}{l}\text { Forma de } \\
\text { aplicação }\end{array}$ & $\begin{array}{c}\mathrm{N}^{\circ} \\
\text { de } \\
\text { Itens }\end{array}$ & Ano & $\begin{array}{l}\text { Língua } \\
\text { original }\end{array}$ \\
\hline $\begin{array}{l}\text { COPPES } \\
\text { Gallagher- } \\
\text { Thompson, } \\
\text { Thompson \& } \\
\text { Rider }\end{array}$ & $\begin{array}{l}\text { Frequência de } \\
\text { envolvimento em } \\
\text { atividades e prazer } \\
\text { subjetivo experimentado } \\
\text { em cada atividade }\end{array}$ & Idosos & Idoso & $\begin{array}{c}\text { Auto- } \\
\text { administrado } \\
/ \\
\text { entrevista }\end{array}$ & 66 & 2004 & Inglês \\
\hline $\begin{array}{l}\text { PES-AD } \\
\text { Teri \& } \\
\text { Logsdon }\end{array}$ & $\begin{array}{l}\text { Frequência, } \\
\text { disponibilidade e prazer } \\
\text { subjetivo experimentado } \\
\text { na realização de } \\
\text { atividades }\end{array}$ & $\begin{array}{l}\text { Idosos com } \\
\text { Alzheimer }\end{array}$ & Cuidador & $\begin{array}{c}\text { Auto- } \\
\text { administrado } \\
/ \\
\text { entrevista }\end{array}$ & 53 & 1991 & Inglês \\
\hline $\begin{array}{l}\text { PAL } \\
\text { Pool }\end{array}$ & $\begin{array}{l}\text { Nível de habilidade do } \\
\text { idoso para se engajar em } \\
\text { atividades que possam } \\
\text { ser prezerosas }\end{array}$ & $\begin{array}{l}\text { Idosos com } \\
\text { Demência }\end{array}$ & Cuidador & $\begin{array}{c}\text { Auto- } \\
\text { administrado } \\
/ \\
\text { entrevista }\end{array}$ & 36 & 1999 & Inglês \\
\hline
\end{tabular}

Observa-se que o COPPES é o único instrumento que não é direcionado para a avaliação de subamostras da população idosa, ao passo que o PES-AD e o PAL destinam-se a avaliar idosos com algum tipo de demência.
As tabelas 2 e 3 resumem os estudos realizados para verificar as propriedades psicométricas de cada instrumento. 
Tabela 2 - Estudos realizados para verificar propriedades psicométricas do COPPES e PES-AD. São Carlos, SP, 2009.

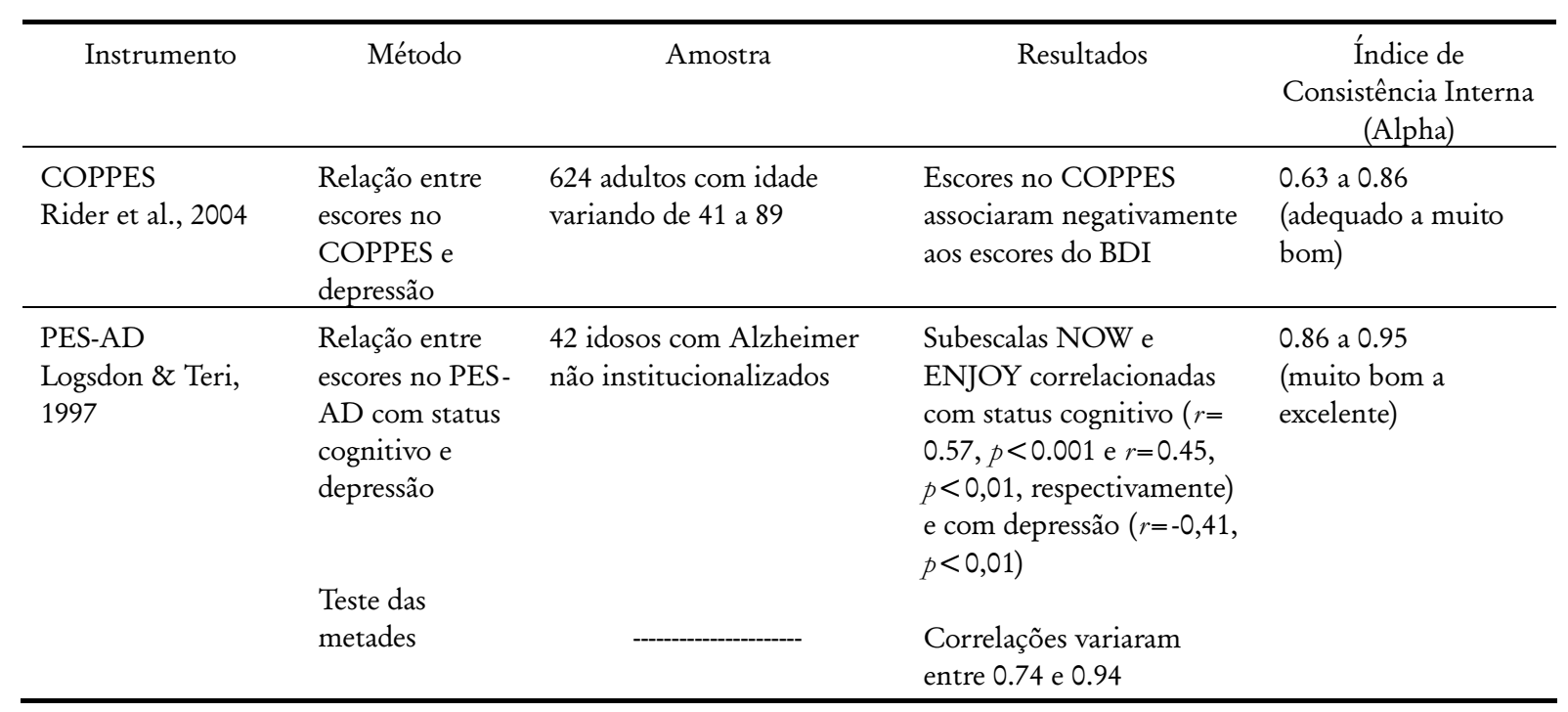

Tabela 3 - Estudos realizados para verificar propriedades psicométricas do PAL. São Carlos, SP, 2009.

\begin{tabular}{|c|c|c|c|c|}
\hline Instrumento & Método & Amostra & Resultados & $\begin{array}{l}\text { Índice de } \\
\text { Consistência } \\
\text { Interna } \\
\text { (Alpha) }\end{array}$ \\
\hline \multirow[t]{4}{*}{$\begin{array}{l}\text { PAL } \\
\text { Wenborn et al., } \\
2008\end{array}$} & $\begin{array}{l}\text { Avaliação da } \\
\text { relevância dos itens e } \\
\text { clareza das instruções } \\
\text { do instrumento }\end{array}$ & $\begin{array}{l}102 \text { profissionais da área de } \\
\text { saúde }\end{array}$ & $\begin{array}{l}97 \% \text { dos profissionais } \\
\text { avaliou as instruções como } \\
\text { sendo claras e } 77 \% \text { avaliou } \\
\text { os itens como sendo muito } \\
\text { importantes }\end{array}$ & $\begin{array}{l}0.95 \\
\text { (excelente) }\end{array}$ \\
\hline & $\begin{array}{l}\text { Relação entre escores } \\
\text { do PAL com status } \\
\text { cognitivo, estado } \\
\text { funcional e grau de } \\
\text { dependência }\end{array}$ & 60 idosos com Demência & $\begin{array}{l}\text { Escores obtidos no PAL } \\
\text { apresentaram relação } \\
\text { significativa com as medidas } \\
\text { avaliadas }\end{array}$ & \\
\hline & $\begin{array}{l}\text { Correlações inter- } \\
\text { item }\end{array}$ & 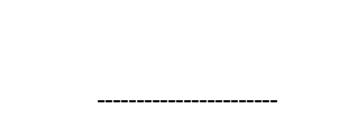 & $\begin{array}{l}\text { A correlação entre grupos } \\
\text { de itens variou de } 0.53 \mathrm{a} \\
0.81 \text {. }\end{array}$ & \\
\hline & $\begin{array}{l}\text { Medidas de teste- } \\
\text { reteste }\end{array}$ & & $\begin{array}{l}\text { Fidedignidade aceitável } \\
\text { através de medidas de teste- } \\
\text { reteste (Coben's Kappa e the } \\
\text { intra class coefficient-ICC) }\end{array}$ & \\
\hline
\end{tabular}

Nota-se que todos os instrumentos apresentam índices de consistência interna que variam de adequado a excelente. O PAL é o instrumento em que foi realizado um maior número de estudos para verificar suas propriedades psicométricas.
No entanto, de forma geral, os estudos realizados para cada instrumento indicam que estes apresentam boas propriedades psicométricas.

Sugere-se que o COPPES seja o instrumento selecionado para ser submetido a um processo de 
validação transcultural no Brasil por algumas razões: (1) na fase inicial de validação de um instrumento, é importante obter uma amostra diversificada para incluir idosos que apresentem níveis variados de envolvimento em atividades prazerosas. Desta forma, é desejável que a amostra de idosos não inclua características específicas (como por exemplo, idosos com demência, que não contam com as habilidades cognitivas necessárias para responder escalas que exigem capacidade de lembrar de forma acurada de eventos do passado recente). O COPPES é um instrumento usado para aferir a frequência de envolvimento de idosos em atividades prazerosas, sem estar direcionado para avaliar especificamente uma subamostra da população idosa (por exemplo, idosos com demência); (2) embora o instrumento tenha sido construído inicialmente para ser utilizado com idosos depressivos em contexto clínico, o COPPES também pode ser utilizado em outros contextos onde seja relevante aferir a frequência de envolvimento em atividades prazerosas do idoso, estando ele depressivo ou não; (3) o instrumento apresenta propriedades psicométricas desejáveis; (4) considera a avaliação subjetiva do idoso sobre a prática de atividades; (5) é de fácil aplicação; e (6) aparenta ser relevante ao contexto brasileiro.

\section{CONCLUSÃO}

Conclui-se que ainda existem poucas pesquisas brasileiras que abordam o envolvimento de idosos em atividades prazerosas, bem como inexistem instrumentos psicométricos brasileiros para aferir este construto. Foram localizados instrumentos na literatura internacional que avaliam a prática de atividades prazerosas por idosos, e o COPPES 5 aparentou ser o instrumento mais apropriado para ser submetido a um processo de validação transcultural para o Brasil, pois se destina a avaliar a prática de atividades prazerosas de idosos, sem ser limitado a uma subamostra da população idosa (por exemplo, idosos com demência).

É importante ressaltar que este estudo apresenta algumas limitações, como: (1) a revisão de literatura foi feita a partir de artigos de livre acesso nas bases de dados acadêmicos consultadas. Isto implica que podem existir outros artigos sobre instrumentos que avaliam o construto em questão, que não foram abordados neste estudo; (2) os instrumentos encontrados são relativamente recentes na literatura, de forma que as evidências de validade não são exaustivas, bem como não foram produzidos muitos estudos utilizando esses instrumentos. Por isso, a utilização desses instrumentos, especialmente no Brasil, deveria acontecer preferencialmente em contextos de pesquisa, com o objetivo de produzir mais informações a respeito das evidências de validade dessas escalas.

Com o crescimento da população idosa no Brasil e diante de dados que apontam que a depressão é o transtorno psiquiátrico mais frequente entre indivíduos com idade avançada ${ }^{30}$, no longo prazo espera-se que uma versão brasileira de um instrumento para avaliar envolvimento de idosos em atividades prazerosas contribua para o planejamento e avaliação de intervenções para tratar depressão em idosos. Além disso, espera-se também que instrumentos desta natureza contribuam para a identificação de estratégias preventivas, focadas na promoção de bem-estar entre os idosos brasileiros. 


\section{REFERÊNCIAS}

1. Lewinsohn PM, Libet J. Pleasant events, activity schedules and depressions. J Abnormal Psychology 1972; 79 (3): 291-295.

2. Bouman TK, Luteijn F. Relations between the pleasant events schedule, depression and other aspects of psychopathology. J of Abnormal Psychology 1986; 95(4): 373-377.

3. Teri L, Logsdon RG. Identifying pleasant activities for alzheimer's disease patients: the pleasant events schedule AD. The Gerontologist 1991; 31 (1): 124-127.

4. Logsdon RG, Teri L. The pleasant events schedule-AD: psychometric properties and relationship to depression and cognition in alzheimer's Disease Patients. The Gerontologist 1997; 37 (1): 40-45.

5. Rider K. California older Person's pleasant events schedule: manual. California :Stanford University; 2004. p.33 . Disponível em: URL: http://www.stanford.edu/group/oafc/Ken/ Manual2.pdf

6. Meeks S, Young CM, Looney SW. Activity participation and affect among nursing home residents: support for a behavioural model of depression. Aging \& Mental Health 2007; 11(6): 751-760.

7. Meeks S, et al. Be activ: a Staff-Assisted Behavioral Intervention for depression in nursing Homes. The Gerontologist 2008; 48 (1): 105-114.

8. Searson R, et al. Activities enjoyed by patients with dementia together with their spouses and psychological morbidity in carers. Aging \& Mental Health 2008; 12 (2): 276-282.

9. Deps VL. Atividade e bem-estar psicológico. In Neri AL. Qualidade de Vida e idade madura. Campinas: Papirus; 1993. p. 57-82.

10. Pondé MP, Caroso C. Lazer como fator de proteção da saúde mental. Rev Ciênc Méd de Campinas 2003; 12 (2): 163-172.

11. Instituto Brasileiro de Geografia e estatística (IBGE). Projeção da população do Brasil. 2008. Disponível em: URL: http://www.ibge.gov.br/ home/presidencia/noticias/ noticia_visualiza.php?id_noticia $=12728$ id_pagina

12. Almeida MMG, et al. Atividades de lazer entre idosos, Feira de Santana, Bahia. Rev Baiana de Saud Pública 2005; 29 (2): 339-352.

13. Lima AMM, Silva HS, Galhardoni R. Envelhecimento bem-sucedido: caminhos para um construto e novas fronteiras. Interface : Comunicação, Saúde, Educação 2008; 12 (27): 795-807.

14. Jenkins KR, Pienta AM, Horgas AL. Activity and health: related quality of life in continuing care retirement communities. Research on Aging 2002; 24(1): 124- 149.

15. Katz S. Busy bodies: activity, aging, and the management of everyday life. J of Aging Studies 2000; 14 (2): 135-152.

16. Florindo AA, et al. Metodologia para avaliação da atividade física habitual em homens com 50 anos ou mais. Rev Saúde Pública 2004; 38 (2): 307-314.

17. Rabacow FM, et al. Questionários de medidas de atividades físicas em idosos. Rev Bras Cineantropometria \& Desempenho Humano 2006; 8 (4): 99-106.

18. Moraes $\mathrm{H}$, et al. $\mathrm{O}$ exercício físico no tratamento da depressão em idosos: revisão sistemática. Rev Psiquiatr 2007; 29 (1): 70-79.

19. Victor JF, Ximenes LB, Almeida PC. Adaptação transcultural para o Brasil da Exercise Benefits/ Barriers Scale (EBBS) para aplicação em idosos: uma avaliação semântica. Cad Saud Pública 2008; 24 (12): 2852-60.

20. Stinson LL. Measuring how people spend their time: a tima-use survey design. Monthly Labor Review 1999; 122(8): 12-19.

21. Schreiner AS, Yamamoto E, Shiotani H. Positive affect among nursing home residents with Alzheimer's dementia: the effect of recreational activity. Aging and Mental Health 2005; 9 (2): 129-134.

22. Doimo LA, Derntl AM, Lago OC. O uso do tempo no cotidiano de mulheres idosas: um método indicador do estilo de vida e grupos populacionais. Cienc Saud Colet 2008; 13 (4): 1133-1142.

23. Gauthier AH, Smeeding TM. Time use at older ages. Research on Aging 2003; 25(3): 247- 274.

24. Neri AL. Qualidade de vida no adulto maduro: interpretações teóricas e evidências de pesquisa. In Neri AL. Qualidade de Vida e idade madura. Campinas: Papirus; 1993. p. 9-55.

25. Havighurst RJ. Older People. Nova Iorque: Mckay; 1953. p.386.

26. Rowe JR, Kahn RL. Successful Aging. Nova Iorque: Pantheon; 1998. p. 265. 
27. Silverstein M, Parker MG. Leisure activities and quality of life among the oldest old in Sweden. Research on Aging 2002; 24(5): 528- 547.

28. Onish J, et al. The pleasurable recreational activities among community-dwelling older adults. Arch Gerontol Geriatr 2006; 43(2): 147-155.

29. Snowdon J. How high is the prevalence of depression in old age? Rev Bras Psiquiatr 2002; 24 (1): $42-47$.

Recebido: $28 / 10 / 2010$

Revisado: $16 / 6 / 2011$

Aprovado: 20/6/2011
30. Baptista MN, et al. Correlação entre sintomatologia depressiva e prática de atividades sociais em idosos. Avaliação Psicológica 2006; 5 (1): 77-85.

31. Wenborn J, et al. Assesing the validity and reliability of the Pool Activity Level (PAL) Checklist for use with older people with dementia. Aging \& Mental Health 2008; 12 (2): 202-211. 